\title{
Attention deficit-hyperactivity symptoms among Palestinian children
}

\author{
A.M. Thabet, ${ }^{1}$ H. Al Ghamdi, ${ }^{2}$ T. Abdulla, ${ }^{1}$ M.-W. Elhelou ${ }^{3}$ and P. Vostanis ${ }^{2}$
}

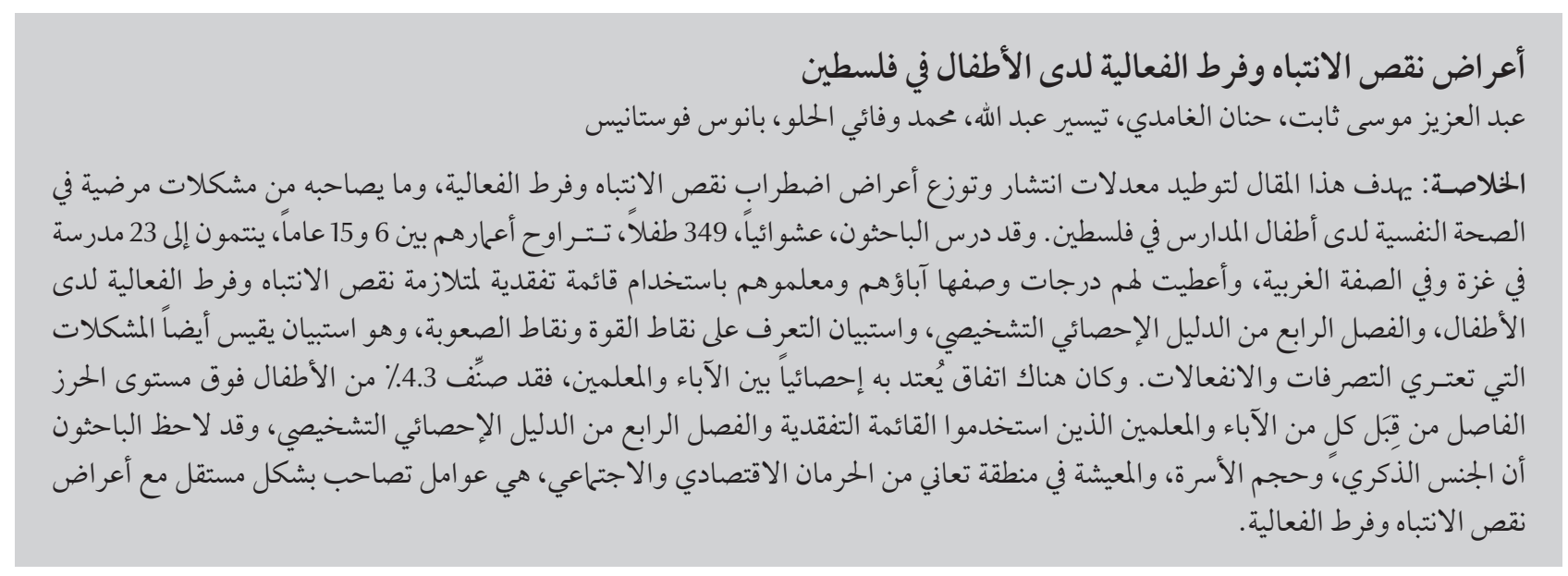

ABSTRACT We aimed to establish the prevalence and distribution of attention deficit-hyperactivity disorder (ADHD) symptoms and other associated comorbid mental health problems in Palestinian schoolchildren Thus 349 children aged 6-15 years were randomly selected from 23 schools in Gaza and the West Bank and were rated by their parents and teachers using both the ADHD DSM-IV Checklist and the Strengths and the Difficulties Questionnaire, which also measures conduct and emotional problems. There was a significant agreement between parents and teachers, with $4.3 \%$ of the children rated above the established cut-off scores on both the parent and teacher DSM-IV Checklist. Male gender, family size and living in an area of socioeconomic deprivation were independently associated with ADHD symptoms.

\section{Symptômes d'hyperactivité avec déficit de l'attention chez les enfants palestiniens}

RÉSUMÉ Notre objectif était d'établir la prévalence et la répartition des symptômes de trouble d'hyperactivité avec déficit de l'attention (THADA) et la comorbidité associée à d'autres problèmes de santé mentale chez les élèves palestiniens. Ainsi, 349 enfants âgés de 6 à 15 ans ont été sélectionnés au hasard dans 23 écoles de Gaza et de Cisjordanie, et évalués par leurs parents et leurs enseignants à l'aide de la liste de contrôle du DSM -IV (4 édition du Manuel diagnostique et statistique des troubles mentaux) pour le THADA et du Strengths and Difficulties Questionnaire (SDQ), qui évalue également les problèmes comportementaux et émotionnels. Une concordance significative a été observée entre les résultats obtenus par les parents et les enseignants, qui ont les uns comme les autres, évalué 4,3\% des enfants au-dessus des seuils définis dans la liste de contrôle du DSM-IV. Le sexe masculin, la taille de la famille et le fait de vivre dans une région de précarité socio-économique sont indépendamment associés aux symptômes de THADA.

${ }^{7}$ Department of Community Mental Health, Al Quds University, Gaza.

${ }^{2}$ Department of Child Psychiatry, University of Leicester, Leicester, United Kingdom (Correspondence to P. Vostanis: pv71@le.ac.uk).

${ }^{3}$ Department of Psychology, Islamic University, Gaza.

Received: 10/05/08; accepted: 15/07/08 


\section{Introduction}

Child mental health problems are increasingly recognised across all societies, with concentrated efforts to improve detection and treatment, through primary health care, schools, welfare and specialist services [1]. Attention deficithyperactivity disorder (ADHD) is a common neurodevelopmental condition of childhood and young life. Its core features include inattention, physical overactivity and impulsivity. In addition, children with ADHD often present with significant psychosocial problems, such as low academic attainment, difficulties with interpersonal relationships, and comorbid oppositional behaviour. Early detection and diagnosis are important in preventing secondary educational and social impairment [2].

The prevalence of ADHD among school-age children varies considerably across different studies and populations [3]. The Diagnostic and statistical manual of mental disorders (4th ed.) reports prevalence rates between $3 \%$ and $7 \%$ for ADHD in the United States (US) [2]. Outside the US, prevalence rates are lower, for reasons that probably reflect diagnostic and methodological differences rather than true prevalence differences [4]. While, there has been limited research on ADHD in developing countries, earlier epidemiological studies suggest variations may be explained by both methodological and cultural differences between ethnic groups even within the same country $[5,6]$.

In recent years, there have been a number of studies on the prevalence, outcome and prognosis of ADHD from populations of Arab children. For example, a study from Qatar estimated that $9.4 \%$ of children had symptoms of clinical significance, i.e. who would merit a clinical assessment, although these did not amount to a clinical diagnosis [7]. When a second stage of diagnostic interviews was included, the real prevalence of $\mathrm{ADHD}$, with associated impairment criteria, was substantially lower, e.g. 1.3\% in Yemen [8] and under $1.0 \%$ in the United Arab Emirates [9,10], which is in line with similarly designed studies [8]. Rates were found to be high, even among female pupils, in Oman, and were associated with aggressive behaviour, school under-achievement and learning difficulties $[11,12]$. High comorbidity rates were found among clinically referred children with ADHD in Saudi Arabia [13].

There have been no reported studies on ADHD in Palestine and therefore we sought to establish rates and the distribution of $\mathrm{ADHD}$ symptoms and other associated comorbid mental health problems in Palestinian schoolage children, as reported by parents and teachers. This will provide an evidencebase for appropriate planning of preventative interventions and treatment programmes.

\section{Methods}

\section{Setting}

The Gaza strip is located between Egypt and Israel, with a population of around 1389789 inhabitants in an area of 360 $\mathrm{km}^{2}$. The West Bank is located to the east of Israel and the west of Jordan, with a population of around 2535927 in $5640 \mathrm{~km}^{2}$ of land. Around $45.9 \%$ of the Palestinian population are under 14 years of age, $50.9 \%$ aged between 15 and 64 years, and only $3.2 \%$ above 65 years. There is a high incidence of unemployment, socioeconomic deprivation, family overcrowding, and short life expectancy [14].

\section{Sample}

A multi-stage random sample design was followed. Schools were randomly selected from a list provided by the Ministry of Education. A random sample of 16 children aged 6-15 years from each of 15 UNRWA (United Nations Refugee and Work Agencies) schools in Gaza was selected; a child in each class year was selected randomly from the class registration book. From the West Bank area, 16 children from each of 8 schools from Bethlehem and East Jerusalem were selected randomly, and were similarly selected from one class registration book.

Permission of the Education Department (UNRWA) to enter the schools to collect data was granted prior to administration of the questionnaires. In addition, the study received approval from the local Ministry of Health research ethical committee.

The data for this study were collected between February and March 2003.

Of the total selected sample $(n=$ 368), parents of 349 children agreed to participate (95\%). A parent (the mother in all cases) signed a consent form that was read to them, outlining the purpose of the study and questionnaire. All measures were administered at the same time. Of the study sample $(n=$ 349), data was not returned by 2 parents and 9 teachers.

\section{Measures}

ADHD-specific rating scales have been found to be reliable and valid for screening and assessing core ADHD symptoms, but these should be supplemented with a clinical interview in order to confirm and establish the diagnosis $[15,16]$. In addition, the use of generic child mental health instruments has been found useful in screening for the frequent comorbid behavioural, emotional and learning $[17,18]$. Therefore to assess the prevalence of ADHD symptoms, we used self-administered and self-rated questionnaires complete by parents and teachers that were based on the following instruments.

Diagnostic and statistical manual IV (DSM-IV) Checklist [19]. This instrument includes 18 DSM-IV ADHD symptoms. Each symptom is scored on a 5-point scale (never, almost never, sometimes, frequently and always), with 
(frequently) and (always) scored as endorsement of positive symptoms. $D S M-I V$ defines 3 subtypes of ADHD; predominantly inattentive, predominantly hyperactive, and combined.

Diagnoses were considered positive if, on the basis of the interview results, DSM-IV criteria were unequivocally met. Children scoring 6 and above on the 9 inattentive items were considered inattentive; children scoring 6 and more on the 9 hyperactivity-impulsive items were considered hyperactiveimpulsive. The combined type is scored by summing both the inattentive and hyperactive-impulsive scores. We prepared an Arabic version of this scale, which was translated and back-translated; the Arabic version was sent to a panel of 5 experts for back-translation. The experts agreed on all items and no changes were made. In our study, for the parents scale, internal consistency was calculated using the Cronbach alpha which was 0.84 ; the split half reliability of the scale was 0.79 . For the teachers scale, internal consistency was $\alpha=0.87$, and the split half reliability was 0.84 .

Strengths and difficulties questionnaire (SDQ) [20]. The SDQ is a well established and widely standardized measure of general mental health problems in childhood. It consists of 25 items, which measure emotional, hyperactivity, conduct and peer problems. The parent and teacher versions of the SDQ were completed. Each item is scored on a $0-2$ scale ( $0=$ not true, $1=$ somewhat true, $2=$ certainly true). A score is estimated for each subscale (range $0-10$ ) with a total difficulties score for the 4 scales in the range of $0-40$, with the exception of prosocial behaviours (positive items), which are not included in the scoring. In our study, Arabic versions of the parent and teacher SDQ were used. These had previously been used in this population $[17,21]$.

Parents were visited at home, and a psychology or social work researcher was available to clarify items while parents completed the questionnaires. If parents were illiterate, the researcher would go through all the questions with them. The participating mothers also provided demographic information on their area of residence, parents' level of education and occupation, family income, and the number of siblings living in household.

\section{Statistical analysis}

We used SPSS, version 13.0 for Windows for data analysis. Descriptive statistics were used to illustrate the demographic characteristic of the sample. Associations between the continuous scores of parents and teachers were established by means of the non-parametric Spearman correlation coefficient test, as the data were not normally distributed.

Stepwise multiple linear regression was used to examine whether any of the sociodemographic variables (sex of child, place of residence, family income and number of siblings) predicted the severity of ADHD symptoms.

\section{Results}

\section{Sociodemographic characteristics}

The sociodemographic characteristics of the sample are shown in Table 1. The mean age of the sample was 10 (range 5-16) years. There were $179(51.3 \%)$ boys and $170(48.7 \%)$ girls. The majority of the children came from the Gaza Strip (57\%). Just over three-quarters of the fathers (77.6\%) were employed, with their type of employment ranging from unskilled labourer to merchant. Almost half of the families (48.1\%) had a low monthly average income of US\$ 300 or less, while $51.9 \%$ were middle to high income families (US\$ 300 to > US\$ 700 monthly). Family size was generally large: $48.2 \%$ of the children had 5-7 siblings and $20.6 \%$ had 8 siblings or more.

\section{Parent and teacher ADHD checklist scores}

When previously established cut-off scores on the ADHD checklist were used, 51 (14.7\%) children were rated above the cut-off by parents and 64 (18.8\%) by teachers, while 15 (4.3\%) of the 341 children with available data, were rated above the cut-off by both parents and teachers.

The sample was small for a sub-analysis on the differences between younger and older children, but no significant correlation was found between age and either parent-reported (Spearman rank correlation $r=-0.069, P=0.20$ ) or teacher-reported $(r=-0.031, P=0.57)$ ADHD scores.

\section{Parent and teacher SDQ scores}

The inter-rater agreement between parent and teacher total and subscale SDQ scores was analysed using the two-tailed Spearman correlation test. There was a significant moderate positive correlation between parents and teachers on total mental health/SDQ scores $\left(r_{s}=\right.$ $0.343, n=339, P<0.0005)$. Moreover, we found a statistically significant moderate correlation between parent and teacher SDQ scores for hyperactivity $\left(r_{\text {s }}\right.$ $=0.380, n=335, P<0.0005)$, conduct $\left(r_{s}=0.347, n=338, P<0.0005\right.$, and emotional scores $\left(r_{\mathrm{s}}=0.328, n=336, P\right.$ $<0.0005$ ).

\section{Parent and teacher ADHD subtype scores}

The relationship between parent and teacher total ADHD combined scores (ADHD-C) was also analysed using the Spearman correlation test. A statistically significant correlation was found $\left(r_{s}=0.26, n=339, P<0.0005\right)$. A statistically significant correlation was also found between parent and teacher scores for predominantly hyperactive (ADHD-H) $\left(r_{\mathrm{s}}=0.255, n=341, P<\right.$ $0.0005)$ and predominantly inattentive items (ADHD-I) $\left(r_{\mathrm{s}}=0.328, n=340, P\right.$ $<0.0005)$. 


\begin{tabular}{|c|c|}
\hline \multicolumn{2}{|l|}{$\begin{array}{l}\text { Table } 1 \text { Sociodemographic } \\
\text { characteristics }(n=349)\end{array}$} \\
\hline Characteristic & No. $(\%)$ \\
\hline Mean age (range) (years) & $10.6(6-15)$ \\
\hline \multicolumn{2}{|l|}{ Sex } \\
\hline Male & $179(51.3)$ \\
\hline Female & $170(48.7)$ \\
\hline \multicolumn{2}{|l|}{ Area of residence } \\
\hline Gaza & $199(57.0)$ \\
\hline West Bank & $150(43.0)$ \\
\hline \multicolumn{2}{|l|}{ Father's employment } \\
\hline Unemployed & $78(22.4)$ \\
\hline Unskilled labourer & 59 (16.9) \\
\hline Skilled worker & $22(6.3)$ \\
\hline Government employee & $156(44.7)$ \\
\hline Merchant & $34(9.7)$ \\
\hline \multicolumn{2}{|l|}{ Family income } \\
\hline Low $^{a}$ & $168(48.1)$ \\
\hline Middle-high ${ }^{\mathrm{b}}$ & $181(51.9)$ \\
\hline \multicolumn{2}{|l|}{ Number of siblings } \\
\hline$\leq 4$ & $109(31.4)$ \\
\hline $5-7$ & $168(48.0)$ \\
\hline$\geq 8$ & $72(20.6)$ \\
\hline
\end{tabular}

${ }^{a}$ US\$300 or less per month; ${ }^{b}$ US\$300->700 per month.

\section{Association between hyperactivity scores on the DSM-IV and SDQ scales}

The parent hyperactivity DSM-IV and SDQ scores were significantly correlated $\left(r_{\mathrm{s}}=0.511, n=345, P<0.0005\right)$. Additionally, a significant correlation was found between the hyperactivity DSM-IV and SDQ scores by the teach$\operatorname{ers}\left(r_{\mathrm{s}}=0.398, n=337, P<0.0005\right)$.

\section{Impact of sociodemographic variables}

Although the etiology of ADHD is well established to be neurodevelopmental, environmental factors can compound the outcome of the disorder, particularly where comorbid mental health problems (predominantly oppositional behaviours) are present. We used stepwise multiple linear regressions to examine which of the sociodemographic variables (independent variables) were associated with the total ADHD DSMIV symptoms scores by both parents and teachers. These variables included child's gender, place of residence, number of siblings and family income.

As in previous studies, male gender was significantly associated with parent total ADHD scores: $F(1,345)=$ $4.45, P=0.036$, adjusted $R^{2}=0.01$. This indicates that $1 \%$ of the variability of ADHD parent scores was accounted for by the child's gender. Additionally, the number of siblings was found to predict parent total ADHD DSM-IV scores: $F(2,344)=4.19, P=0.016$, adjusted $R^{2}=0.018$. This indicates that nearly $1.8 \%$ of ADHD parent scores were accounted for by family size. The regression model was repeated using total ADHD DSM-IV teacher scores, but no statistically significant associations were found.

When total child mental health (SDQ) scores, as rated by parents, were entered as the dependent variable in a subsequent stepwise regression analysis, area of residence was significantly associated: $F(1,343)=8.17, P=0.005$. Parent-rated scores in Gaza were significantly higher than in the West Bank. The adjusted $R^{2}(0.021)$ indicated that about $2 \%$ of the variability of total SDQ parent scores was accounted for by area of residence, which is a marker of socioeconomic deprivation. The regression analysis was repeated, with each of parent SDQ subscale score, as the dependent variable. Area of residence was significantly associated with hyperactivity $\left(P<0.0005\right.$, adjusted $R^{2}$ $=0.1)$ and conduct scores $(P<0.001$, adjusted $\left.R^{2}=0.03\right)$. A similar association was established between the area of residence and teacher total mental health SDQ scores: $F(1,335)=$ $11.868, P<0.001$, adjusted $R^{2}=0.03$; hyperactivity $\left(P=0.02\right.$, adjusted $R^{2}=$ $0.13)$, and conduct scores $(P<0.0005$, adjusted $R^{2}=0.06$ ).

\section{Discussion}

Our findings indicate that the prevalence ADHD symptoms that justify a clinical assessment and other more impairing disorders that require treatment, is higher than previously thought. Based on screening questionnaires or rating scales, previous studies have reported prevalence rates between 5\% and 9\% $[7,22,23]$, i.e. similar to the findings of this study. These children do not usually suffer from a disorder - the clinical rates for disorders are much lower, closer to $1 \%-2 \%[8]$ - but still require skilled observation and monitoring from their teachers and health professionals, such as paediatricians or nurses. At the same time this sub-clinical threshold is still important for the identification of clinical cases (in our study, parent and teacher scores indicated that $15 \%$ and $18 \%$ of the children respectively would be clinical cases) because such screening could help identify high-risk children.

The high estimate of ADHD symptoms with our questionnaire possibly resulted from the use of less stringent criteria, without corroboration between informants, clinical observations and inclusion of impairment criteria [24]. The translation and the linguistic meaning of the diagnostic criteria and parents' and teachers' perceptions of the meaning of disruptive behaviour may have also contributed to the high estimate. This is an important issue for future research across different cultures and populations; comparative studies are needed so that findings can be interpreted in a similar context.

An important implication of the overall findings is the need to train teachers and primary health care professionals to be able to recognize the symptoms of ADHD and seek specialist assessment for the more severe cases [25].

Our study ascertained moderate agreement between parents and 
teachers using 2 standardized rating scales. This is consistent with previous studies $[25,26]$. Discrepancies in the ratings reported by adult informants have been attributed to several factors, such as the child's changing behavior in different situations or settings, and different ratings being based on adults subjective views [27]. Obtaining information from teachers is sometimes difficult as teachers may not be available during school vacations or because children have multiple teachers at higher grades. Different ratings may also be attributed to parents being more tolerant of disruptive behaviour and unwilling to report problems to avoid stigmatization. For these reasons, it is important that clinicians corroborate information from both sources.

Although ADHD has a clearly established neurodevelopmental etiology, various psychosocial adversities such as socioeconomic deprivation, family conflict and maternal psychopathology have been found to increase the risk for ADHD and associated morbidity, and these factors have a cumulative effect [28]. On examining whether the sociodemographic status contributed to ADHD and the related behavioural problems in our study, we took into consideration the variables of family size, family income and area of residence. Large family size, coming from areas of higher deprivation and male gender had a contributing effect, although this was partly explained by their association with behavioural/conduct problems.

Our study has certain limitations. The findings relied on informant rating score of children's ADHD symptoms and other co-occurring problems, without being supplemented with clinical interviews to confirm the diagnosis. This may have resulted in an overestimate of the prevalence of ADHD symptoms. Another concern related to the administration of the 2 rating instruments in a standard meaningful way to avoid judgmental views and over-reliance on subjective adult views. In addition, the sociodemographic status variables used in this study were limited and did not include wider psychosocial adversity measures, such as parental discord or mental illness, and acute and chronic life events. The measures used in this study require cross-cultural validation before being widely applied in future research. Future research should aim to compare prevalence rates across different age groups.

In spite of the limitations, this was one of the first studies to measure ADHD symptoms in a large representative Arab child population, and based on 2 types of adult informants and 2 research instruments. These baseline findings can thus form a useful guide for educating parents and training teachers and primary health care professionals in the early detection of likely cases who may require specialist assessment and treatment.

\section{Acknowledgement}

We are grateful to all parents and teachers who took part in this study.

\section{References}

1. Vostanis P. National Service Framework: Its principles are relevant to all countries. Child \& Adolescent Mental Health, 2005, 10: $131-2$

2. American Academy of Paediatrics, Clinical Practice Guideline: Diagnosis and evaluation of the child with attention-deficit/ hyperactivity disorder. Journal of the American Academy of Paediatrics, 2000, 105:1158-70.

3. Taylor E et al. European clinical guidelines for hyperkinetic disorder: First upgrade. European child \& adolescent psychiatry 2004, 13 (Suppl. 1):1730.

4. Karnik N, Dogra N, Vostanis P. Child psychiatry across cultures. In: Bhugra D, Bhui K, eds. Textbook of cultural psychiatry. Cambridge, Cambridge University Press, 2007:471-83.

5. Livingstone R. Cultural issues in diagnosis and treatment of ADHD. Journal of the American Academy of Child \& Adolescent Psychiatry, 1999, 38:1591-4.

6. Bird H. Epidemiology of childhood disorders in a cross-cultural context. Journal of child psychology \& psychiatry, 1996, 37:5-49.

7. Bener A, Qahtani R, Abdelaal I. The prevalence of ADHD among primary school children in an Arabian society. Journal of attention disorders, 2006, 10:77-82.

8. Alyahri A, Goodman R. The prevalence of DSM-IV psychiatric disorders among 7-10-year-old Yemeni schoolchildren. Social psychiatry \& psychiatric epidemiology, 2008, 43:224-30.

9. Eapen $\mathrm{V}$ et al. Mental health problems among schoolchildren in United Arab Emirates: Prevalence and risk factors. Journal of the American Academy of Child \& Adolescent Psychiatry, 1998, 37:880-6.

10. Eapen V, Jakka ME, Abou-Saleh M. Children with psychiatric disorders: The Al Ain community psychiatric survey. Canadian journal of psychiatry, 2003, 48:402-7.

11. Al-Sharbati $\mathrm{M}$ et al. Attention deficit-hyperactivity disorder in a sample of Omani schoolboys. Canadian journal of psychiatry, 2004, 49:572.

12. Al-Sharbati $M$ et al. Attention deficit-hyperactivity disorder in Omani schoolgirls. Journal of the American Academy of Child \& Adolescent Psychiatry, 2004, 43:132-3.

13. La-Haidar FA. Co-morbidity and treatment of attention deficit - hyperactivity disorder in Saudi Arabia. Eastern Mediterranean health journal, 2003, 9:988-95.

14. Palestinian National Authority. The Status of Health in Palestine: 2005 Annual Report. Gaza, Ministry of Health, 2005.

15. Barkley R. Attention deficit-hyperactivity disorder: A handbookfor diagnosis and treatment. New York, Guilford Press, 2006.

16. Brown M. Diagnosis and treatment of children and adolescents with attention-deficit/hyperactivity disorder. Journal of counselling \& development, 2000, 78:195-200.

17. Thabet AA, Stretch D, Vostanis P. Child mental health problems in Arab children: Application of the Strengths and Difficulties Questionnaire. International journal of social psychiatry, 2000, 46:266-80. 
18. Goodman R et al. Using the Strengths and Difficulties Questionnaire (SDQ) to screen for child psychiatric disorders in a community sample. British journal of psychiatry, 2000, 177:534-9.

19. American Psychiatric Association. Diagnostic and Statistical Manual of Mental Disorders, 4th edition. Washington DC, American Psychiatric Association, 2004.

20. Goodman R. Psychometric properties of the Strengths and Difficulties Questionnaire (SDQ). Journal of the American Academy of Child and Adolescent Psychiatry, 2001, 40:1337-45.

21. Alyahri A, Goodman R. Validation of the Arabic Strengths and Difficulties Questionnaire and the Development and WellBeing Assessment. Eastern Mediterranean health journal, 2006, 12(Suppl. 2):S138-46.

22. Ersan E et al. The distribution of symptoms of attention-deficit/ hyperactivity disorder and oppositional defiant disorder in school age children in Turkey. European child \& adolescent psychiatry, 20004, 13:354-61.

23. Gadow $\mathrm{K}$ et al. Comparison of attention deficit-hyperactivity disorder symptom subtypes in Ukrainian schoolchildren. Journal of the American Academy of Child \& Adolescent Psychiatry, 2000, 39:1520-7.
24. Wolraich M et al. Examination of DSM-IV criteria for attention deficit-hyperactivity disorder in a county-wide sample. Journal of developmental \& behavioral pediatrics, 1998, 19:162-8.

25. Wolraich $\mathrm{M}$ et al. Teachers' screening for attention deficithyperactivity disorder: Comparing multinational samples on teacher ratings of ADHD. Journal of abnormal child psychology, 2003, 31:445-55.

26. De Nijs P et al. Attention deficit - hyperactivity disorder (ADHD): Parents' judgment about school, teachers' judgment about home. European child \& adolescent psychiatry, 2004, 13:315-20.

27. Biederman J et al. How informative are parent reports of attention deficit-hyperactivity disorder symptoms for assessing outcome in clinical trials of long-acting treatments? A pooled analysis of parents' and teachers' reports. Pediatrics, 2004, 113:1667-71.

28. Biederman J, Faraone S, Monuteaux M. Differential effect of environmental adversity by gender: Rutter's Index of Adversity in a group of boys and girls with and without ADHD. American journal of psychiatry, 2002, 159:1556-62.

\section{Facts on mental health}

About half of mental disorders begin before the age of 14 years. Around 20\% of the world's children and adolescents are estimated to have mental disorders or problems, with similar types of disorders being reported across cultures. Yet, regions of the world with the highest percentage of population under the age of 19 have the poorest level of mental health resources. Most low- and middle-income countries have only one child psychiatrist for every 1 to 4 million people.

War and other major disaster have a large impact on the mental health and psychosocial well-being. Rates of mental disorder tend to double after emergencies.

Source: WHO Fact file: 10 facts on mental health: (http://www.whoint/features/factfiles/mental_health/en/index.html\#). 